\title{
BUCANEROS EN EL ESTRECHO DE MAGALLANES DURANTE LA SEGUNDA MITAD DEL SIGLO XVII, NUEVOS ANTECEDENTES
}

MATEO MARTINIC B. ${ }^{\text {a }}$

\section{RESUMEN}

El paso de filibusteros por el estrecho de Magallanes durante la segunda mitad del siglo XVII es un hecho del acontecer histórico regional conocido en general, pero sobre el que persisten incógnitas en lo tocante a la frecuencia de pasos y a lo acontecido durante sus misteriosas travesías. En el artículo se aportan nuevas informaciones sobre la presencia de bucaneros franceses en las últimas décadas de la centuria; se describe un mapa hasta ahora desconocido cuya autoría se les atribuye y se hacen consideraciones acerca del papel determinante que tuvieron los mismos, por el conocimiento que tuvieron del territorio marítimo del Estrecho y sus experiencias de vida y relación con los nativos del área, en los ulteriores proyectos oficiales del gobierno de Luís XIV para establecer una colonia en Magallanes y, además, en el principio y desarrollo del comercio de contrabando de naves francesas con los dominios españoles de Chile y de Perú.

PALABRAS CLAVE: Bucaneros, estrecho de Magallanes, cartografía, Patagonia.

\section{BUCCANEERS IN THE STRAIT OF MAGELLAN DURING THE SECOND HALF OF THE XVII CENTURY, NEW INFORMATION ABSTRACT}

The passage of buccaneers through the Strait of Magellan during the second half of the seventeenth century is a fact of general historical and regional knowledge. Nevertheless uncertainties persist in regard to the frequency of trips and what happened during these mysterious journeys. New information concerning the presence of French filibusters in the last decades of the century is provided in this article. We describe an hitherto unknown map whose authorship is attributed to these buccaneers and other considerations are relative to the decisive role they played, their knowledge of the maritime territory of the Strait of Magellan and life experiences with relation to native groups of the area. This, during the later period of official government projects by Louis XIV, when they tried to establish a colony in Magallanes and also they begin and develop smuggling trade in French ships within the Spanish dominions of Chile and Peru.

KEY WORDS: Buccaneers, Strait of Magellan, chartography, Patagonia.

a Profesor Emérito, investigador Centro de Estudios del Hombre Austral, Instituto de la Patagonia, Universidad de Magallanes,

Punta Arenas. mateo.martinic@umag.cl 


\section{INTRODUCCIÓN}

La presencia de naves extrañas en el estrecho de Magallanes durante el siglo XVII, bien de banderas nacionales conocidas (españolas, holandesas, inglesas, francesas) o de pabellones piratas es una materia que todavía no está satisfactoriamente resuelta en cuanto a información disponible. Bien conocida en lo que se refiere el primer quinto y al último lustro de la centuria, dista de serlo en lo que se refiere al extenso lapso intermedio. En efecto, está bien documentada la presencia de naves de bandera holandesa y española entre 1599 y 1619 , la inglesa de 1669-70 y las francesas de 1696 a 1700 , según lo dado a conocer con anterioridad (principalmente Martinic, 1992 (2006) y 1999), pero es evidente que los lapsos intermedios aún se mantienen en la penumbra. No obstante que se poseen noticias sobre lo que en ellos pudo acontecer, las mismas son imprecisas, como, a vía de ejemplo, las entregadas por Francisco de Seixas de Lovera (1690) en las que no hay certidumbre acerca del rumbo seguido por los barcos que pasaron desde el Atlántico al Pacífico y viceversa, esto es, si lo hicieron por la ruta del Estrecho o por la más recientemente conocida del cabo de Hornos, que parece haber sido lo probable.

Comprendemos ahora mejor la razón (o una de las razones) de la ausencia de las naves de las potencias marítimas, España, Holanda, Inglaterra y Francia, al enterarnos de las turbulencias políticas, sociales, bélicas y climáticas que sacudieron a Europa durante el siglo que ha sido denominado "maldito" por algunos historiadores y que exigieron en sus respectivos casos atender con prioridad otros asuntos domésticos o internacionales más importantes que las navegaciones ultramarinas por el remoto meridión de América. El complejo suceder $e$ interacción de acontecimientos que convulsionaron especialmente al Viejo Mundo, con múltiples consecuencias ha sido estudiado entre otros académicos, por Geoffrey Parker (2013).

La preocupación académica por la materia de que se trata, los viajes transfretanos, es de antigua data y recurrente en el tiempo hasta nuestros días, con resultado de la elaboración de un registro de viajes que se considera tan completo como para pensar que es muy difícil agregar alguna nueva información de interés sobre el asunto.

Sin embargo de lo investigado y publicado desde largo tiempo atrás, ello se ha referido principalmente a la expediciones documentadas, pero debe aceptarse que en lo conocido hasta el presente permanecen algunas lagunas o vacíos de información que podrían corresponder a viajes con insuficiente o ninguna cobertura documental, por lo que únicamente el azar o la casualidad brindan la posibilidad de aumentar en el curso de nuevas pesquisas las noticias disponibles. Tales podrían ser los casos de alguna misteriosa travesía clandestina, intencionadamente mantenida oculta para evitar el control oficial hispano, y los no menos ocultos periplos de navíos piratas.

Como haya sido, lo que ha podido averiguarse da cuenta de una interrupción en la secuencia informativa prácticamente total entre 1619 y 1669, con apenas algunos registros ocasionales con posterioridad y hasta promediar la década final del siglo XVII. Pero, no debiera caber duda acerca de la posibilidad de ocurrencia de alguna travesía misteriosa aun no registrada documentalmente y recordamos para el caso, como sugerente indicio, la referencia que encontramos dibujada en el mapa manuscrito de John Narborough del estrecho de Magallanes, que da cuenta del hallazgo de anclas en la costa septentrional de la Primea Angostura de este canal, como señal evidente del paso de algún desconocido navío antes de que lo hiciera el navegante inglés.

Así entonces, tratamos de llenar en parte el vacío informativo añadiendo a lo conocido nuevos datos que pacientemente hemos compulsado a lo largo de varios años.

\section{LA PRESENCIA DE BUCANEROS FRANCESES Y SUS CONSECUENCIAS}

Hasta ahora teníamos por cierto que el francés Jean Baptiste de la Feuillade y el inglés Bartolomé Sharp, ambos filibusteros, el primero en 1667 y el segundo en 1682 fueron los primeros navegantes que estuvieron en las zonas occidentales del Estrecho, aquél de manera comprobada como que naufragó en un paraje desconocido del gran canal, y Sharp del que se sabe que intentó ingresar al Estrecho, aunque fue en vano. Otras noticias han permitido agregar el paso de los corsarios ingleses 
John Eaton y Basil Ringrose en 1684 y de bucaneros franceses que al parecer lo hicieron hacia 16871694. Se trataba de antiguos piratas del Caribe que tras la campaña represiva que se realizó en su contra, principalmente por las autoridades inglesas de Jamaica, y que virtualmente puso término a esa perniciosa actividad en las aguas de aquel vasto mar interior, buscaron seguridad cruzando el istmo de Panamá y navegaron por el Pacífico sudamericano y prosiguieron con sus correrías. Dueños de un rico botín, se concentraron finalmente en las islas Juan Fernández (Más a Tierra) enterando una escuadrilla de cinco naves. Repartido el tesoro que habían acumulado, cada buque optó por un camino propio para retornar a Europa. Los bucaneros franceses que tripulaban un barco de doscientas toneladas eligieron la vía del estrecho de Magallanes. Una vez aquí, si bien consiguieron ingresar por la zona occidental del gran canal, acabaron naufragando según parece sobre la costa de la actual bahía Galiano, en la península Ulloa de la isla Santa Inés, sector que los bucaneros llamaron canal de Joucqjoucq recogiendo e interpretando la denominación indígena (kawéskar) para el mismo. Aunque su nave fue lanzada por la fuerza del mar hacia la costa rocosa y por ello quedó severamente averiada, no se hundió, lo que permitió que los náufragos pudieron aprovechar sus restos con los que construyeron una especie de bergatín de unas catorce toneladas, faena laboriosa que exigió una permanencia alrededor de once meses ${ }^{1}$. Ese tiempo fue empleado además en la caza y pesca para procurarse alimentos y otros recursos, $y$ en la exploración de las aguas y litorales circundantes, actividad en la que pudieron relacionarse y establecer un amistoso trato con los kawéskar, canoeros nómades que habitaban desde hacía milenios el archipiélago occidental de la Patagonia y la parte correspondiente de la Tierra del Fuego, desde el golfo de Penas hasta el canal Brecknock. Concluida

1 Al parecer el astillero improvisado se levantó en la bahía Batchelor, costa continental del Brunswick, del otro lado de la bahía Galiano y al norte de la isla Carlos III. Allí excavaciones preliminares recientes en busca de sitios indígenas de poblamiento antiguo, han revelado la presencia de restos culturales de origen europeo tales como metales, vidrios y monedas francesas que sugieren la posibilidad de un asentamiento de circunstancias (comunicación personal Dra. Dominique Legoupil).

2 Journal du voyage fait a la mer du Sud avec les filibustiers su labor de construcción zarparon Estrecho avante hacía el Atlántico llevando consigo, además de su experiencia de vida harto dura por las obligadas privaciones y por la inclemencia climática, una noción siquiera aproximada acerca del laberinto marino en el que habían permanecido. Luego de algunas recaladas para fines de reabastecimiento los antiguos bucaneros llegaron a Francia hacia 1688. De cuantos pudieron integrar el grupo se conocen los nombres de Ravenau de Lussan, que publicó en 1689 una crónica de su notable aventura ${ }^{2}$ y unos tales Massertie (Macerty o Mac Carthy), de la Marre y Jouhan de la Guilbaudière.

Tras una incursión, varios años después, en 1690, arribó al estrecho de Magallanes el filibustero inglés John Strong, con su navío Wellfare y que navegó el paso procediendo del Atlántico y con rumbo al Pacífico con sucesivas recaladas que demoraron su estadía entre febrero y mayo, y luego en el viaje de retorno a Europa en el curso de diciembre de aquel año. Esta navegación tuvo transcendencia para las ciencias naturales y por ello ha sido reconocida en los anales históricos ${ }^{3}$.

Por largo tiempo se tuvo por cierto que tanto esta navegación como la accidentada precedente de los franceses habían sido las únicas incursiones marítimas del género en aguas interiores de Magallanes, pero ahora sabemos de otra sobre la que nos ha dejado testimonio un tal Gregorio de Robles, su forzado protagonista. Este, español de origen, se embarcó como soldado en el navío La Perla que zarpó de Cádiz en 1688 con destino a San Agustín, Florida. De allí luego de dejar el servicio real dio comienzo a una vida aventurera de viajero que lo llevó por Cuba, Jamaica, Nueva Granada y Perú, desde donde retornó a las Antillas. Capturado por filibusteros franceses cuando se iniciaba 1694, navegó con ellos hasta el sur de África, continuó hacia Europa, Lisboa, y de allí pasó a Brasil, siguiendo el periplo por la costa

\section{d'Amerique en 1684 et année suivantes, 1689.}

3 En efecto, embarcado en la nave inglesa iba el médico cirujano John Sloane, quien como parte de sus tareas colectó especímenes botánicos en el litoral del Estrecho $y$ que posteriormente, con otros materiales naturales permitió organizar el afamado herbario del Museo Británico (Cfr. Hugo Gunckel, "Las primeras plantas herborizadas en Chile en 1690", Anales del Instituto de la Patagonia, II (1-2), 134-145, 1971. 
sudamericana oriental rumbo al sur, arribando a la boca oriental del estrecho de Magallanes hacia el mes de septiembre, canal por el que navegaron adentro en procura del paso al Pacífico.

Sobre lo que fuera esa virtualmente desconocida incursión interesa ceder la palabra al propio Robles, que nos da cuenta de ella en breve y precisa cita:

Desde Puerto Deseado, porel término de 18 días, fue entrando hasta cavo Galán, que será más de las dos tercias partes del estrecho y lo más angosto, respecto de que desde él se ven las montañas del Reyno de Chile, y se descubren el bolcán de la Tierra de que llaman del Fuego. [Se] desmontó una pieza de artillería, púsola en tierra y experimentó que, disparando desde la banda superior, atravesava y con bentaja llegava a la otra, curiosidad que descubría sus intentos. Pero no pudo pasar adelante, porque le sobrevino un recio temporal con densa neblina, nieve y bentisca tal que quitava la vista a la gente, siendo esto por el mes de septiembre, con que les fue preciso desandar lo andado y volverse a Puerto Deseado, como lo ejecutaron en cinco días, porque el viento Sur lo facilitava entonces ${ }^{4}$.

Nada dice Robles sobre quienes fueron sus captores, ni el nombre de la nave en que uno y otros viajaban, pero no cabe dudar de que el tracto relatado corresponde a una navegación en el Estrecho. En efecto, los datos que en la breve narración se contienen son precisos y claros: el cabo Gallant, al que corresponde la mención, es un accidente exterior de la bahía Fortescue, antepuerto del fondeadero Gallant, usualmente entendido como un conjunto; su situación en la extensión del litoral del Estrecho corresponde, millas más o menos, con los dos tercios de su desarrollo; en su demanda se avista el conspicuo monte Sarmiento al que este célebre navegante nombrara "Volcán Nevado"; el canal interoceánico, por otra parte, prosigue en estrechura a partir de los pasos

4 Gregorio de Robles, América a fines del siglo XVII. Noticias de los lugares de contrabando. Casa-Museo de Colón. Seminario Americanista de la universidad de
Tortuoso, Largo y del Mar hacia el noroeste, y, por fin, la mención a la rudeza e impredictibilidad climática (en la época) en ese sector del curso fretano era cosa sabida de los navegantes desde el descubridor Fernando de Magallanes y no fueron pocos los que no lograron pasar adelante, rumbo del Pacífico, por más esfuerzos que hicieron.

Queremos abundar sobre la referencia implícita a puerto Gallant, tempranamente considerado por los marinos como uno de los mejores fondeaderos del estrecho de Magallanes. Así, haber alcanzado hasta alli -es nuestra hipótesisfue un arribo programado a base de alguna información conocida por los filibusteros. Estos, por ello, si no eran compinches de los protagonistas del naufragio de marras, podían haber obtenido de los mismos, siquiera de modo indirecto, noticias sobre aquel sector interno de mar magallánico que les aseguraba un buen tenedero, recursos variados $y$, con suerte, nativos pacíficos con los cuales en buen trato podían manejarse. Así, persiste la duda acerca de quiénes fueron los captores de Robles y, con él, navegadores del Estrecho.

¿Hubo otras incursiones filibusteras por este gran canal durante el tercio final del siglo XVII fuera de las ya registradas? Creemos que tal posibilidad no puede desecharse, más si hay indicios que permiten sostenerla. En efecto, además de la misteriosa presencia de anclas en el litoral de la Primera Angostura consignada cartográficamente por John Narborough, cabe recordar como sugerente evidencia arqueológica la estructura de piedra que se sitúa en la caleta Steamer, en el indicio de la costa septentrional de la punta de Santa Ana (península Brunswick) y en inmediata vecindad del lugar en que Pedro Sarmiento de Gamboa fundó la Ciudad del Rey Don Felipe en 1584. Por sus características esa construcción ubicada en un lugar apropiado por demás para varar y reparar una nave, parece haber servido como horno de circunstancia para alguna faena de reparación naval, forja o aderezado de piezas de hierro tal vez, y cuya fecha de construcción se ha estimado hacia 1630, con un margen de variación de treinta años ${ }^{5}$.

Valladolid. Valladolid 1980, 43-44.

5 Véase del autor "Ciudad del Rey Don Felipe (Estrecho de Magallanes, Chile). Consideraciones acerca de su defensa. Magallania, 31, 5-13. 2003. 
Nada, absolutamente nada, se sabe acerca de quién pudo hacerlo y sobre sus razones para ello. Pero quien haya sido su misterioso constructor, es claro que debió ser un navegante foráneo que posiblemente tuvo una recalada forzosa y que encontró un lugar muy apropiado para reparar su embarcación.

Habrá que seguir investigando, pues, tanto en lo referido a este asunto particular, como a la incógnita respecto de otras travesías que pudieron tener ocurrencia en el estrecho de Magallanes entre 1619 y 1696.

\section{UN DESCONOCIDO MAPA MANUSCRITO DEL ESTRECHO DE MAGALLANES}

Una década atrás, poco más o menos, Charles Porter, ingeniero norteamericano asociado a la Universidad de Magallanes en algunas investigaciones referidas a los ambientes archipielágicos de la región, nos obsequió una reproducción facsimilar (fotocopia en blanco $y$ negro) de un curioso mapa que muestra la parte meridional de la antigua Magallania. La copia obtenida al parecer en la Biblioteca del Congreso de los Estados Unidos de América reproduce un mapa manuscrito cuyo título reza DESTROIT DU MAGELLAN ET DE SES ENVIRONS. A la vista de un conocedor, de primera la representación contenida en el plano nos recordaba a las bien conocidas cartas holandesas del estrecho de Magallanes de las primeras décadas del siglo XVII, especialmente las debidas a Pieter Keer y Willem Blaeu, pero se trata solo de una impresión de bulto y contornos que desaparece al observarse en detalle.

Hoy sabemos más sobre esta curiosa y prácticamente desconocida pieza gracias al estudio particular hecho por Anthony Mullan, especialista en Bellas Artes de la División Ciencias Sociales de la Biblioteca del Congreso de los Estados Unidos de América, publicado hace algunos años ${ }^{6}$. La misma integra un extenso manuscrito titulado Description des principaux endroits de la mer du Sud depuis les 52 degrez 30 minutes Sud, ouest le d'Estroit du Magelland Jusqu 'au 41, degrez Nord, qui est liisle de Californie, compuesto por un texto

6 Jouhan de la Guilbaudière, his "Buccaneer's Atlas", and the Beginnings of Franch trade along the Pacific Coast of descriptivo -derrotero- que abarca buena parte del litoral occidental americano desde la península de California a la boca del estrecho de Magallanes en Chile, complementado con 35 mapas y planos. $\mathrm{Su}$ autor es nuestro ya conocido Jouhan de la Guilbaudière y, de acuerdo con Mullan, se trata de un escrito preparado para interesar a algún importante armador de Saint Malo (probablemente Noël Danycan de L`Epine) o, quizá, confeccionado a su expresa petición. No sabemos cómo este documento, único al parecer, llegó hasta el famoso repositorio librario y documental norteamericano, en cuyo inventario figura incorporado bajo el título "Jouhan de la Guilbaudière Buccaneer's Atlas" (Geography and Map Division).

El objetivo, es claro, era el de brindar una información fiable y actualizada para la navegación en las -para los franceses de la época- desconocidas costas occidentales de América, hasta las que podían extenderse los intereses mercantiles sensu lato, esto es, desde el contrabando al mercadeo lícito, asunto que por entonces generaba un gran interés en la Francia de Luis XIV.

Centrándonos en las ilustraciones de la Descripción, en los mapas propiamente, su autor fue el Señor de la Hanche, al que Guilbaudière recurrió para el efecto. Sin desconocer su competencia para llenar un cargo calificado como era el que investía, Ingeniero del Rey, sí consideramos que sus dotes como dibujante cartográfico eran mediocres a juzgar por la pieza de que se trata que, dicho sea de paso, responde en su ejecución al estilo ilustrativo del género para la época en Francia. En su diseño, el mismo se ajustó a las instrucciones de Buchotte, un ilustrador especialista, que para entonces regulaban la producción cartográfica en cuanto al colorido usado para identificar y diferenciar planos orográficos (llanuras pastosas, colinas boscosas, montañas nevadas); las aguas marinas y terrestres, y otros aspectos notorios naturales; y las señales para identificar ciudades, poblaciones y fortalezas.

La organización del conjunto documental, descripciones y mapas, fue pensada para ofrecer un recorrido informativo norte-sur y su ordenamiento corresponde a las diferentes noticias obtenidas (robadas o secuestradas y copiadas) de los españoles, y a las observaciones practicadas

Routh America. 1700. The PORTOLAN, Journal of the Washington Map Society, 75,17-31, 2009. 


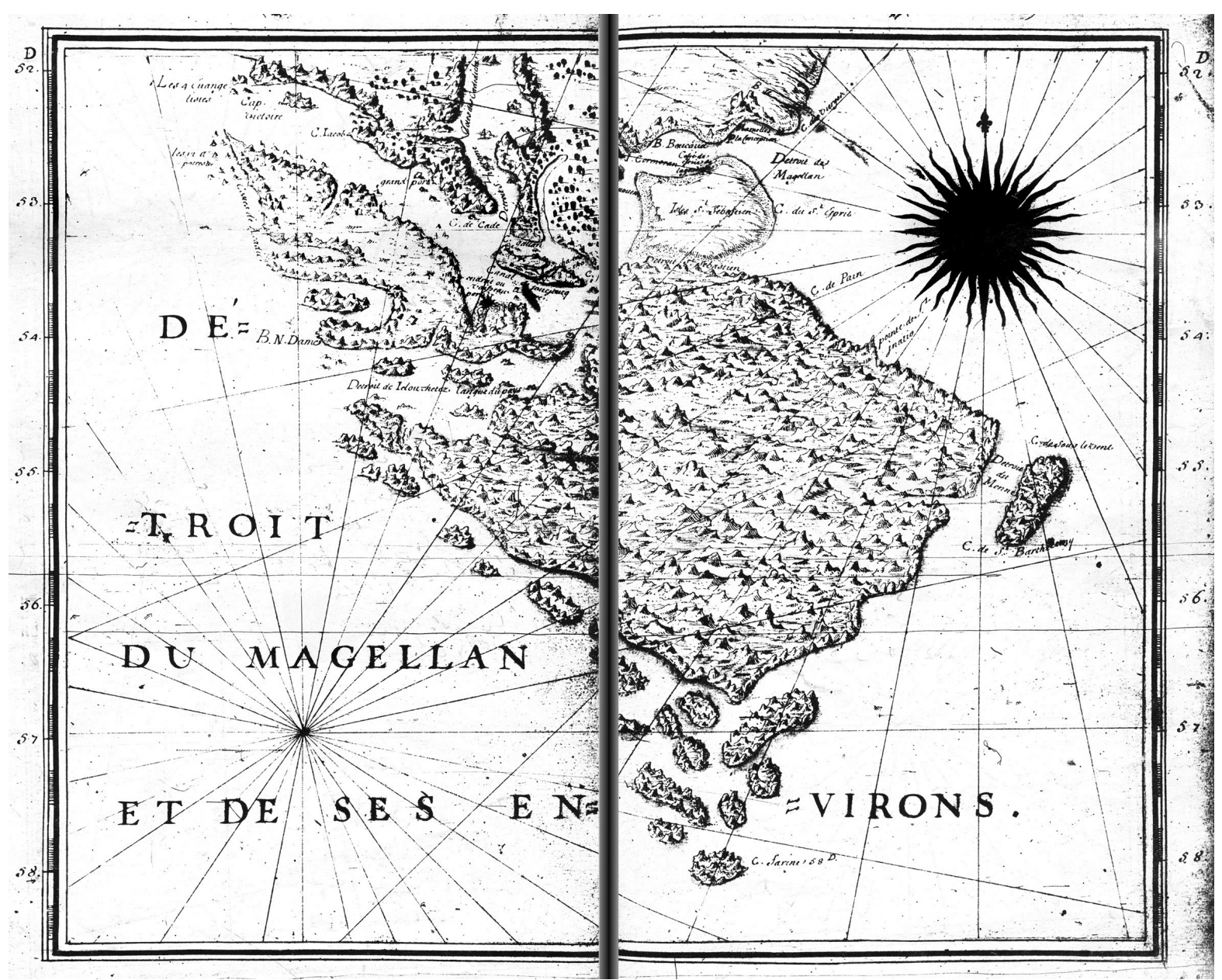

Fig. 1. Déstroit du Magellan et de ses environs (Facsiml reducido).

por los propios bucaneros franceses en el curso de sus travesías predatorias.

El original de la pieza de que se trata, está definido en sus bordes por unas líneas que lo enmarcan, mide $35 \mathrm{cms}$ de ancho por $28 \mathrm{cms}$ de alto; en los costados el marco tiene graduada la latitud y en la parte externa de ambos lado, de arriba abajo se observan las letras $D$ y las cifras $52,53,54,55,56,57,58$ que corresponden a los grados. El título y los topónimos están en francés. La pieza en descripción, reiteramos, no se parece a ningún otro plano del área previamente conocido, excepción hecha de la Carte du Detroit de Magellan, mapa manuscrito elaborado por el antiguo filibustero Johan de la Guilbaudière en 1697 y que se conserva en el Departement de Cartes et Plans, Bibliotheque Nationale, Paris, que dimos a conocer hace tiempo en nuestra Cartografía Magallánica 1523-19457. La semejanza se da en la toponimia de origen indígena y en algunas formas geográficas, según se comenta más adelante.

El territorio cartografiado corresponde a la parte meridional de la Patagonia desde el océano Atlántico al Pacífico, dividida en su parte central, con sentido norte-sur, por un canal ancho y al que en su sector austral se denomina Detroit s. Hieronyme. Al sur del estrecho de Magallanes, bien figurado en su desarrollo natural del noreste, al suroeste, sur y noroeste según sus inflexiones y rumbos conocidos, la Tierra del Fuego es representada dividida en tres partes compactas: al norte, la Isle S. Sebastien que está separada por el canal homónimo que corre del 
Estrecho al Atlántico, de un gran sección territorial cuadrangular cuyo interior se muestra lleno de montañas. Al suroeste del estrecho de Magallanes y al oeste de la porción insular mayor mencionada hay una gran isla que se extiende desde la boca occidental al centro del Estrecho. Ambas islas principales están separadas por el Detroit de Idouchetes. Una quincena de islas menores se adosan a la isla mayor por su costado sudoccidental y se extienden como archipiélago al sur de la misma. Por el este figura sin identificar únicamente la actual isla de los Estados. Por fin, junto a la tierra occidental se muestran otras cuatro islas pequeñas, de la que la más importante es aquella situada dentro de Estrecho, entre Patagonia y Tierra del Fuego y a la que no se da nombre. La misma está separada de la isla occidental fueguina por el canal de Joucq joucq y de la parte patagónica por un canal sin nombre, en cuya parte continental se lee C. Gallant.

La semejanza con el mapa de Guilbaudière mencionado (del que difiere en la figuración de las islas fueguinas mayores, dos en aquella $y$ tres en éste), se hace notoria en la toponimia, particularmente en la de origen vernáculo que ambas recogen.

He aquí el detalle de acuerdo a nuestra sectorización geográfica:

Litoral pacífico: Les evangelistas, les 12 apotres, Cotes de Acadie, B.N. Dame, Detroit de Iduchetes Langue du pays.

Parte occidental del Estrecho: Cap Victorie, C. Iacob, grand port, $C$ de Cade, Detroit s. Hieronyme, Cotes des Cadediches.

Parte central del Estrecho: C. Gallant, (ilegible) che (ilegible) cho, C. Fruart.

Parte oriental del Estrecho: (ilegible) gnoven, I. Cormoren, Cote de (ilegible) laguediches, B. Boucand, Isles St. Sebastien, B. de Magnelles, P. La Conception, C. De Vierges, Detroit de Magellan.

8 En la copia utilizada para esta descripción el mapa presenta en su parte central, de arriba abajo, una línea de sombra por efecto de la apertura forzada a que se sometió el volumen en
Costa atlántica fueguina: C. Du St. Esprit, C de Pain, Pointe de St. Inatio, Detroit de Menne, C. De Sous le vent, C. De Barthelemy, C. Sarine [o Farine?], 58 D.

La graduación latitudinal consignada tanto por el este como por el oeste es idéntica: D 52, $53,54,55,56,57,58$. Como exorno adicional, el dibujante del mapa incluye una rosa de los vientos (que más parece un sol), desde la que salen líneas de rumbos hacia los puntos cardinales (loxodrómicas), una de las cuales tiene puesta una flor de lis que marca el norte 8 (Fig. 1).

Sin embargo del rudo estilo del dibujante, hay en la pieza en descripción un afán ornamental que se manifiesta en las formas orográficas y en la vegetación, siguiendo un diseño que recuerda cercanamente a los mapas fretanos del mismo siglo elaborados por los maestros holandeses Willem Blaeu (1631) y Pieter Keer (1628-1645). La parte sudoriental de Patagonia es mostrada sin montañas para aparentar su carácter orográficamente más llano, excepto la singular formación volcánica, visible en el paisaje nororiental del Estrecho, lo que sugiere una observación de viaje (Monte Aymond y Orejas del Burro actuales). La península de Brunswick se muestra arbolada y más atractiva a la vista según la apreciaron los navegantes de la época. Los detalles que sorprenden son los de la desmesurada penetración que se da a puerto Gallant, figurándolo como un profundísimo fiordo, por una parte, y por otra el desconocimiento del topónimo "Cabo de Hornos" suficientemente conocido por los navegantes y cartógrafos del siglo XVII, que se reemplaza por el extraño nombre de Sarine o Farine.

La intervención que indudablemente cupo a Guilbaudière en el plano surge no sólo de su comparación con otro mapa cuya autoría se le reconoce, a lo que nos referimos a continuación, sino de la particular mención que se hace del lugar del siniestro de la nave bucanera en la bahía Galiano, según se ha visto antes, puntualizada con la precisa anotación junto a la costa de la isla occidental del mapa: endroit ou vaisseau peris, referencia de ocurrencia cronológicamente situada entre 1684 y 1690.

que se incluye que, evidentemente, esta encuadernado. En la reproducción que acompañamos se ha procurado bajar la tonalidad de la sombra. 
Ahora bien, en una correlación con el mapa mencionado de Guilbaudière, Carte de Detroit de Magellan, que pudimos observar años atrás durante nuestra visita al Departamento de Cartas y Mapas del Archivo Nacional de París, y que registramos en nuestro Catálogo bajo el número $146^{9}$ y que atribuimos con duda al antiguo bucanero, cabe considerar la precedencia de elaboración de ambas piezas, esto es, aceptando que fueron contemporáneas o si fueron sucesivas o simultáneas. Si lo primero, es de interés determinar cuál lo fue antes o, de otro modo, si la primera en trabajarse pudo servir de modelo a la segunda. Por otra parte es difícil aceptar una simultaneidad sugerida por el estilo del dibujo cartográfico, la coincidencia de algunos topónimos vernáculos y de algunas menciones expresas de acontecimientos a la vista de diferencias de figuración territorial lo que, mejor pensado, sugiere más que simultaneidad sensu strictu una proximidad temporal en la elaboración, previa revisión de bocetos elaborados in situ, de memoria al cabo de incursiones exploratorias de reconocimiento por parte de los náufragos de marras. En cualquier caso, no debiera excluirse el cotejo con o siquiera la observación paralela con algún anterior mapa holandés del Estrecho. Pero, así y todo, resulta difícil de comprender una representación de conformación geográfica tan irreal -pocas islas de gran tamaño con litorales parejos, en vez de un piélago laberíntico como lo es verdaderamente el sector occidental magallánico- y, por ello, tan diferente del que hoy conocemos. Pieza interesante por demás la presentada y descrita, tanto por sus defectos, como por sus equívocos y sugerencias.

Finalmente, si se hace una comparación entre los planos de Magallania elaborados por los filibusteros ingleses (Sharp, Ringrose) y los bucaneros franceses (Guilbaudière y tal vez algún otro), respondiendo por igual unos y otros al estilo de dibujo cartográfico más bien burdo y tosco, modalidad a la que también se ajusta la producción hispana contemporánea, los de procedencia gala con todo lo fantasiosos son más detallados y con mejor aproximación a la realidad geográfica territorial conocida actualmente. Su mérito principal estriba en que, sin embargo de sus errores, los

9 Cfr. Cartografía Magallánica 1523-1945 (Ediciones de la Universidad de Magallanes, Punta Arenas 1999), mismos sirvieron de fundamento para la posterior elaboración de los mapas de Magallanes por los ingenieros De Labat y Duplessis, integrantes de la expedición de Beauchesne-Gouin en 1699.

\section{CONCLUSIONES}

$\mathrm{Si}$ bien persiste la incógnita sobre la posibilidad de alguna travesía por el estrecho de Magallanes hasta ahora desconocida, especialmente en lo que se refiere al medio siglo corrido entre el viaje de los hermanos Nodal (1619) y la expedición de John Narborough (1669-1670), se ilumina más el conocimiento de lo allí ocurrido entre 1670 y 1695 con la recurrente presencia de filibusteros y bucaneros.

A estos, a los franceses en especial, deben acreditarse dos hechos determinantes: uno, la información hidrográfica y etnográfica aportada con su simple y algo rústica cartografía que fue obtenida al cabo de varias incursiones exploratorias $y$, todo así lo hace parecer, de pacífico trato con los indígena con los cuales fue maravilla que de alguna manera los bucaneros pudieron entenderse como para informarse sobre las parcialidades etnográficas y topónimos identificatorios de accidentes geográficos, hecho cuyo único precedente lo hallamos en el viaje realizado un siglo antes por Pedro Sarmiento de Gamboa. Esta información, interesante de suyo, fue recibida y reelaborada por especialistas de la Marina Francesa y aumentada con la producción subsiguiente originada en los sucesivos viajes de naves de la armada real, con lo que se configura todo un ciclo de aportes galos en la historia cartográfica de Magallanes y Chile.

En los mismos, además, está el origen de una sucesión de acontecimientos concatenados entre sí de relevancia en la historia nacional como fueran la incursiones filibusteras sobre la costa central del antiguo Reino y del Virreinato del Perú, con la noción acerca de las posibilidades para el ejercicio mercantil; la accidentada travesía de retorno a Europa de un grupo de esos bucaneros que tanto fueron portadores de aquella noticia como de la recomendación de fundar un establecimiento de Francia en la zona interior del estrecho de Magallanes como eventual base operativa para futuras acciones

Catálogo, pág. 104. 
marítimas-mercantiles; el subsiguiente interés oficial en la materia expresado en expediciones conducidas por Jean Baptiste de Gennes (16951697) y por Jacques de Beauchesne-Gouin (16981700) que aunque fallidas en su objetivo inicial la instalación de una colonia en puerto Gallant o sus inmediaciones- ${ }^{10}$, permitieron abrir el océano Pacífico, en particular los litorales de Chile y Perú, al comercio de contrabando que, con altibajos, se mantuvo por el siguiente cuarto de siglo hasta 1724 , con inesperadas y duraderas consecuencias políticas, sociales y culturales para la Capitanía General de Chile según ha sido investigado y estudiado por diversos historiadores, entre otros por Diego Barros Arana (1886), Francisco A. Encina (1949), Fernando Campos Harriet (1964), Sergio Villalobos (1986) y Armando Cartes Montory (2004).

Nos ha llamado la atención el hecho de que atendida la prolongada presencia naval francesa (bucanera entre 1684-1695, y oficial entre 16951701) en el estrecho de Magallanes, alguna noticia de ello no hubiera llegado al conocimiento de las autoridades de Chiloé -vía tradición oral indígena-, moviéndolas en consecuencia a investigar acerca de su efectividad, como sucediera en su hora y con comprensible revuelo con la presencia holandesa y posteriormente con el paso de Narborough, con las expediciones de reconocimiento encomendadas a Jerónimo Díez de Mendoza (1674) y Antonio de Vea (1675-76).

Al parecer esas autoridades habían quedado "curadas de espanto" luego de las fabulaciones de Talcapillán ${ }^{11}$, o quizá, el mentado "correo indio" no había resultado tan eficaz, si de ello puede escribirse en este caso. Como hubiera sido, no se sabe de ninguna reacción sobre la presencia de los filibusteros y bucaneros fuera esta de las autoridades locales o nacionales ante esa ocasional circunstancia en las aguas magallánicas del Reino de Chile. Lo único que se conoce es una reacción tardía del gobernador Francisco Ibáñez de Peralta (1701) motivada más, al parecer, por la reciente y recurrente presencia francesa en el

10 Es posible que el gobierno de Francia abandonase, siquiera de momento, la idea de una colonia en el Estrecho en consideración a la nueva situación política generada en Europa tras la muerte de Carlos II, el último monarca de la casa de Austria y el acceso al trono de España del nieto del mapa manuscrito de la Región Magallánica, obra de Francisco de Seixas y Lovera (1690) que en su parte superior izquierda, a la altura de la actual isla Hanover, expresa Fortaleza (y un dibujo de casas). Aquí están poblados los Yngleses dede el año 1675. Con este pueblo y algunas (ilegible) en la costa, mención que debe entenderse motivada por la recalada patagónica del capitán filibustero inglés Bartolomé Sharp en 1682 o más propiamente por la cartografía elaborada por su compañero de correrías Basil Ringrose, cuya publicación posterior debió causar alguna preocupación en el gobierno de España.

Este desconocimiento acerca de lo que sucedía en el distante territorio austral chileno es un asunto que debiera investigarse por los historiadores contemporáneos para, en definitiva, hacer más luz sobre la noción real que las autoridades de Chile del período de entre siglos XVII y XVIII tenían sobre su remota jurisdicción magallánica.

\section{AGRADECIMIENTOS}

El autor deja constancia de su agradecimiento para con el extinto Charles Porter que entregó la interesante información cartográfica referida a la matería, así como para el antropólogo Joaquín Bascopé Julio y para el arqueólogo Alfredo Prieto Iglesias por su búsqueda y colaboración en la obtención de información adicional sobre algunos tópicos de este trabajo.

\section{FUENTES DE CONSULTA}

Hanisch, W. (1982). La Isla de Chiloé, capitanía de rutas australes. Academia Superior de Ciencias Pedagógicas: Santiago.

Howse, D., \& Norman J. W. T. (Eds.). (1984). A Buccaneer's Atlas Basil Ringrose's South Sea Waggoner. BerkeleyLos Angeles-Oxford: University of California Press.

Malamud, C. (1986). Cadiz y Saint Malo en el comercio colonial peruano (1698-1725). S/E.

Martinic, M. (1999). Cartografía Magallanica 1523-1945. Ediciones de la Universidad de Magallanes: Punta

rey Luis XIV, el futuro Felipe V.

11 Véase al respecto de María X. Urbina "El Chono Cristóbal Talcapillán y su información sobre colonias inglesas en la Patagonia Insular, 1674 (Boletín de la Academia de Historia Naval y Marítima de Chile, Valparaíso, 2016). 
Arenas.

Martinic, M. (2003). Ciudad del Rey Don Felipe (Estrecho de Magallanes, Chile). Consideraciones acerca de su defensa. Magallania, 31(1), 5-13.

Martinic, M. (2006[1992]). Historia de la Región Magallanica: Tomo I. Ediciones de la Universidad de Magallanes: Punta Arenas.

Martinic, M. (2011). El occidente fueguino. Todavía una incógnita. Gasco Magallanes: Punta Arenas.

Mullan, A. (2009). Jouhan de la Guilbaudière his "Buccaneer's Atlas", $y$ and the Beginnings of French Trade along the Pacific Coast of America ca. 1700. The Portolan Journal of the Washington Map Society. Issue 75 Fall 2009: 17-31. Washington D.C.
Parker, G. (2013). El siglo Maldito. Clima, Guerras y Catástrofes en el siglo XVII. Planeta: Barcelona

Robles, G. (1980). América a fines del siglo XVII. Noticias de los buques de contrabando. Casa-museo Colón. Seminario Americanista de la Universidad de Valladolid. Valladolid.

Seixas y Lovera, F. (1690). Descripción Geográphica y Derrotero de la Región Austral Magallanica. Madrid.

Urbina, R. (1983). La periferia meridional indiana. Chiloé en el siglo XVII. Ediciones universitarias de ValparaísoUniversidad Católica de Valparaíso: Valparaíso.

Vásquez de Acuña, I. (2004). Historia Naval del Reino de Chile 1520-1826. Síntesis de Ana Victoria Durruty Corral. Edición del autor: Santiago. 